\title{
Efficiently generate functional hepatic cells from human pluripotent stem cells by complete small- molecule strategy
}

\section{Tingcai Pan}

Southern Medical University

\section{Ning Wang}

Guangzhou Institutes of Biomedicine and Health

Jiaye Zhang

Guangzhou Institutes of Biomedicine and Health

\section{Fan Yang}

Jinan University

\section{Yan Chen}

Guangzhou Institutes of Biomedicine and Health

\section{Yuanqi Zhuang}

Guangzhou Institutes of Biomedicine and Health

\section{Yingying Xu}

Guangzhou Institutes of Biomedicine and Health

Ji Fang

Guangzhou Institutes of Biomedicine and Health

\section{Kai You}

Guangzhou Institutes of Biomedicine and Health

Xianhua Lin

Guangzhou Institutes of Biomedicine and Health

\section{Yang Li}

Southern Medical University

\section{Shao Li}

Southern Medical University

\section{Kangyan Liang}

Southern Medical University

\section{Yin-xiong Li}

Guangzhou Institutes of Biomedicine and Health

Yi Gao ( $\nabla$ gaoyi@smu.edu.cn )

Southern Medical University 


\section{Research Article}

Keywords: Hepatic differentiation, small molecule, human pluripotent stem cells, hepatoblasts, hepatocyte-like cells

Posted Date: December 22nd, 2021

DOI: https://doi.org/10.21203/rs.3.rs-1169294/v1

License: (c) (i) This work is licensed under a Creative Commons Attribution 4.0 International License.

Read Full License

Version of Record: A version of this preprint was published at Stem Cell Research \&amp; Therapy on April 11th, 2022. See the published version at https://doi.org/10.1186/s13287-022-02831-1. 


\section{Abstract}

Background: Various methods have been developed to generate hepatic cells from human pluripotent stem cells (hPSCs) that rely on the combined use of multiple expensive growth factors, limiting industrialscale production and widespread applications. Small molecules offer an attractive alternative to growth factors for producing hepatic cells since they are more economical and relatively stable.

Methods: We dissect small-molecule combinations and identify the ideal cocktails to achieve an optimally efficient and cost-effective strategy for hepatic cells differentiation, expansion, and maturation.

Results: We demonstrated that small-molecule cocktail CIP efficiently induced definitive endoderm (DE) formation via increased endogenous TGF- $\beta$ /Nodal signaling. Furthermore, we identified that combining Vitamin C, Dihexa, and Forskolin (VDF) could substitute growth factors to induce hepatic specification. The obtained hepatoblasts (HBs) could subsequently expand and mature into functional hepatocyte-like cells (HLCs) by the established chemical formulas. Thus, we established a stepwise strategy with complete small molecules for efficiently producing scalable HBs and functionally matured HLCs. The small-molecule derived HLCs displayed typical functional characteristics as mature hepatocytes in vitro and repopulating injured liver in vivo.

Conclusion: Our current small-molecule based hepatic generation protocol presents an efficient and costeffective platform for the large-scale production of functional human hepatic cells for cell-based therapy and drug discovery using.

\section{Background}

Developing efficient and cost-effective technology to generate large quantities and high-quality functional human hepatic cells is essential for applying hepatic cells to cell-based therapy and drug toxicity screening. To date, protocols have been developed to generate hepatic cells from hPSCs, while most of the reported protocols relied on expensive growth factors, such as Activin A, BMPs, FGFs, and HGF [1-5]. This leads to critical challenges such as high cost and low reproducibility. In contrast, small molecules offer an attractive alternative to growth factors since they are usually more economical, relatively more stable, and possibly more efficient for generating hepatic cells from hPSCs [6-8]. Thus, substituting growth factors with small molecules and optimizing the hepatic generation method could contribute to scalable and integrated producing functional hepatic cells, improving cost-effectiveness and reproducibility.

Previous efforts have been made to identify small molecules that support the differentiation and maintenance of hepatic cells, and impressive advances have been undertaken to identify numerous chemical candidates [9-11]. Our previous study established a synergistic cocktail to regulate BMP, Wnt, Hedgehog, and other signaling pathways, creating a delicate balance for HBs expansion and bipotency maintenance [12]. Based on this, we further created a robust and cost-effective culture condition for the generation of self-renewing HBs and functional mature HLCs, using a small-molecule cocktail in a defined 
culture system [13]. Further use of a small-molecule protocol to direct hPSCs differentiation into HBs, and establish a complete small-molecule formula to generate expanded HBs and functional mature HLCs efficiently would benefit many hepatic cell potential clinical applications, including cell transplantation, bio-artificial liver, drug development, and disease modeling.

So far, complete small-molecule derived HLCs protocols have been reported in several works of literature, with a lower hepatic differentiated efficiency than growth-factor based approaches [6, 14-16]. This may be due to the indistinct contribution of exogenous and endogenous signals involved in hepatic differentiation. More importantly, these studies are generally characterized by hepatic marker genes and proteins expression and albumin secretion and glycogen uptake while lacking hepatocyte-specific functional analyses in vitro and in vivo. Thus, a more detailed understanding of the precise regulatory mechanisms of hepatic differentiation is vital to establishing a small-molecule based induction protocol. Also, further efforts are needed to optimize the current chemical differentiation procedure for efficient and robust differentiation hPSCs into functional hepatic cells.

In the present study, we focused on improving chemical strategy to generate functional hepatic cells from hPSCs. We demonstrated that stepwise treatment with small molecules, including CHIR99021 (CHIR), IDE1 and PD0332991 (PD), could elevate endogenous TGF- $\beta$ /Nodal signaling activity and direct hPSCs toward definitive endoderm (DE) efficiently. We further screened small molecules to identify an optimal chemical condition VDF for HBs specification. Moreover, the small-molecule derived HBs could subsequently expand and mature into HLCs via our previously established chemical cocktail. The smallmolecule derived HLCs showed typical morphologic and functional features of mature hepatocytes, with hepatocyte-specific markers expression and functionalities in vitro and in vivo. In summary, our current chemical-based hepatic specification, expansion, and maturation method presents an efficient and robust platform for the cost-effective and large-scale production of functional human hepatic cells for clinical regenerative medicine and drug discovery applications.

\section{Material And Methods}

\section{Human PSCs culture and hepatic differentiation}

Human PSC lines, including iPSC line (UC15) and ESC line (H1) were cultured in mTeSR1 medium (Stem Cell Technologies) on 100-fold-diluted Matrigel matrix (Growth Factor Reduced, BD Bioscience). The hPSCs colonies were passaged using Accutase (life).

For hepatic differentiation, we started DE induction first. Briefly, while hPSCs reached nearly $70 \%$ confluence, the medium was replaced with RPMI1640 (Gibco) supply with 1×B27 (minus insulin, Invitrogen), containing CHIR99021 (CHIR), IDE1, $2 \mu \mathrm{M}$ Ly294002 (Ly), and $0.75 \mu \mathrm{M}$ PD0332991 (PD) as indicated in Fig. $1 \mathrm{~A}$ and Fig. $2 \mathrm{~A}$ for 3 days. Further DE specifying to $\mathrm{HBs}$, DE cells were cultured in differentiation medium (RPMI1640 supply with 1×B27 supplement [Invitrogen]), containing growth 
factors (20 ng/mL BMP2 and $30 \mathrm{ng} / \mathrm{mL}$ FGF4) or small molecules (10 $\mu \mathrm{g} / \mathrm{mL}$ Vitamin C, $0.1 \mu \mathrm{M}$ Dihexa, and $10 \mu \mathrm{M}$ Forskolin) as indicated in Fig. 4A.

\section{Human HBs culture and hepatic maturation}

HBs were maintained in expansion medium as described previously [12]. Briefly, the purified HBs were cultured in expansion basal medium (RPMI1640, 1× B27 supplement, 1× ITS) supplemented with chemical cocktails (ACDFSV), including $5 \mu \mathrm{M}$ A8301, $3 \mu \mathrm{M}$ CHIR99021, $0.1 \mu \mathrm{M}$ Dihexa, $10 \mu \mathrm{M}$ Forskolin, $0.5 \mu \mathrm{M}$ SAG, and $10 \mu \mathrm{g} / \mathrm{mL}$ Vc. Used chemical compounds were purchased from Selleck except indicated. For cell replating in our current method, the expanding HBs were dissociated by Accutase (life) and replated on Matrigel Matrix pre-coated plates.

For hepatocyte maturation, HBs were cultured in hepatoZYME-SFM (Gibco) medium supply with $1 \mathrm{X}$ GlutaMAX, containing small-molecules, including $5 \mu \mathrm{M}$ A8301, $0.1 \mu \mathrm{M}$ dexamethasone (Dex), $0.1 \mu \mathrm{M}$ Dihexa and $0.5 \mathrm{mM} \mathrm{NH4Cl}$ for 5 days. The medium was changed daily during the differentiation period. Used small molecules were purchased from Selleck, and growth factors were purchased from PeproTech except that indicate.

\section{Functional analyses of differentiated hepatocyte in vitro}

For the urea secretion assay, the culture media of 24 hours incubated in differentiated cells were collected and stored at $-80^{\circ} \mathrm{C}$. Urea concentration was analyzed by LC/MS/MS API3000 and normalized with total cell protein concentration.

To evaluate the CYP450 activity, cultures were incubated with conventional probe substrates (CYP3A4: 6 $\mu \mathrm{M}$ midazolam, CYP2C9: $10 \mu \mathrm{M}$ diclofenan, CYP2D6: $10 \mu \mathrm{M}$ dextromethorphan) respectively, for quantifying metabolite production. After 2 hours of exposure, the culture medium was collected and stored at $-80^{\circ} \mathrm{C}$ subsequently, and CYP450 activity was analyzed by LC/MS/MS API3000. Metabolite products were normalized to total cell protein.

To evaluate the glycogen production and storage ability, Periodic acid-Schiff (PAS) staining was performed. According to the manufacturer's instructions, the cultured cells were fixed with $4 \%$ PFA for 30 minutes, and intracellular glycogen was stained using a PAS staining solution (Muto Pure Chemicals).

For cellular indocyanine green (ICG) uptake assay, differentiated cells were incubated in media supplemented with ICG (final concentration of $1 \mathrm{mg} / \mathrm{ml}$, Sigma-Aldrich) for 1 hour at $37^{\circ} \mathrm{C}$. Cells were then washed with PBS and imaged using a phase-contrast microscope (X71, Olympus).

For cellular LDL uptake assay, the HLCs were cultured with a medium containing Alexa-Flour 488-ac-LDL for $1 \mathrm{~h}$, and immunohistochemistry was performed. Nuclei were counterstained with DAPI.

\section{Animal model and hepatic cells transplantation}


Immune-deficient NOD-SCID-IL2RG ${ }^{-/-}$mice (NSI mice, GIBH) were used as recipients of human hepatic cells. Before hepatic cells transplantation, 8-week-old NSI mice received DMN intraperitoneal injections (7 $\mathrm{mg} / \mathrm{kg}$, Sigma, $1.0 \%$ dissolved in saline) for 2 consecutive days for inducing acute liver injury. Two days later, $1 \times 10^{6}$ hepatic cells were intrasplenically transplanted into the DMN-treated NSI mice. To monitor the transplantation state, recipient mice livers were harvested at different time points after hepatic cells transplantation. All animal experiments were approved by the Animal Welfare Committee of GIBH. All protocols were approved by the appropriate institutional animal care and use committee (IACUC).

\section{Quantitative RT-PCR analysis}

According to the manufacturer's protocol, total RNA was extracted using TRIzol reagent (Invitrogen) and quantified with NanoDrop 2000 (Thermo Fisher). cDNA was reverse transcribed from $2 \mu \mathrm{g}$ RNA using ReverTra Ace (Toyobo) and oligo-dT (Takara). Quantitative RT-PCR was performed with the CFX96 machine (Bio-Rad) and SYBR Green Premix (Bio-Rad) following the manufactures' manual. The GAPDH was used for quantitative RT-PCR normalization, and the experiments have repeated a minimum of three times to confirm the results. Primer sequences are listed in the Supporting information Table 1.

\section{Immunohistochemistry staining}

Cells were washed with PBS and fixed with 4\% PFA (Sigma-Aldrich) for $30 \mathrm{~min}$ at room temperature. After washing the cells with PBS, cells were permeabilized with $0.1 \%$ Triton X-100 in PBS for 30 minutes and blocked with PBS containing $5 \%$ normal goat or donkey serum for 30 minutes at room temperature. The primary antibodies were diluted with blocking solution and incubated at $4^{\circ} \mathrm{C}$ for overnight. After washing the cells with PBS, cells were then stained with compatible Alexa Fluor-conjugated secondary antibodies

in blocking solution for $1 \mathrm{~h}$ at room temperature. Nucleus stained with $5 \mu \mathrm{g} / \mathrm{mL}$ DAPI (Invitrogen). Imaging was performed on a Zeiss LSM 710 confocal microscope. The primary antibodies and secondary antibodies were described in the Supporting information Table 2.

\section{Fluorescence-activated cell sorting (FACS) and flow cytometry analysis}

Cells were dissociated with Accutase and then resuspended in PBS containing 3\% BSA. The collected cell suspensions were stained with FITC-conjugated human Ep-CAM antibody (Milteny) and Alexa Fluor APCconjugated antibody against human C-Kit (B56; BD Biosciences) for 30 minutes on ice. EpCAM ${ }^{+} / \mathrm{C}^{-\mathrm{Kit}^{-}}$ cells were sorted using a MoFloTM fluorescence-activated cell sorter. Sorted cells were seeded on Matrigel pre-coated plates, and maintained in expansion basal medium containing small-molecule cocktails.

To analyze the cell proliferation, the expanded HBs were dissociated and then resuspended in PBS containing $10 \%$ FBS. The collected cell suspensions were fixed with $4 \%$ PFA (Sigma-Aldrich), and incubated in blocking and permeabilizing buffer, containing $0.1 \%$ Triton X-100, and $5 \%$ normal donkey serum in PBS for 30 minutes at room temperature. The cells were then incubated with APC-conjugated Ki67 (B56; BD Biosciences) and other indicated antibody for 30 minutes on ice. Corresponding isotype 
antibodies were used as controls. Flow cytometry analyses were performed using a FACS Aria II flow cytometer (BD Biosciences).

\section{Statistical analysis}

The data were analyzed with Sigma Plot 10.0. Statistical differences between two groups were tested with a two-tailed Student's t-test. Data are represented as mean \pm SEM. Survival data were analyzed with the Kaplan-Meier test. For all tests, ${ }^{*} \mathrm{p}<0.05$ was considered significant.

\section{Results}

\section{CHIR and IDE1 inefficiently induced DE differentiation from hPSCs}

Our previous studies and other reports have demonstrated that CHIR, a GSK-3 $\beta$ inhibitor that acts as a Wnt agonist, combined with Activin A could induce hPSCs efficient differentiation into DE [12, 17-19]. However, Activin A is an expensive growth factor, limiting its use in large-scale cell production. In order to replace Activin A, researchers have tried to screen available small molecules and found that IDE1 and IDE2 are the only two molecules that could partly replace Activin A for DE induction [7, 20-22]. Thus, we examined if IDE1 could replace Activin A, combined with CHIR, to direct DE differentiation efficiently, according to the Activin A plus CHIR treated pattern (CA) (Fig. 1A).

Our results showed that this small-molecule cocktail (Cl-I) failed to induce homogeneous DE-like cells morphology as CA (Fig. S1). The immunostaining showed that the $\mathrm{Cl}-\mathrm{I}$ merely yielded 70\% SOX17 positive cells, and plenty of cells remained OCT4 positive or appeared to express BRA, indicating mixed undifferentiated hPSCs and mesoderm (MD) cells (Fig. 1B). Therefore, we further optimized the smallmolecule cocktail by supplying $1 \mu \mathrm{M} \mathrm{CHIR}$ on day 2 and $3(\mathrm{Cl})$. The results showed that the optimized condition improved DE induction slightly, but still tricky to derive DE efficiently as CA formula (Fig. 1B).

Moreover, genes expressions of pluripotency, Primitive streak (PS), primitive endoderm (PrE), and DE were dynamically traced daily during the differentiation process (Fig. 1C). The results showed a minor decrease in the pluripotency markers OCT4 and NANOG expression levels. In contrast, DE markers SOX17 and FOXA2 increased lower in cells treated with $\mathrm{Cl}-\mathrm{I}$ or $\mathrm{Cl}$ condition, compared with $\mathrm{CA}$ treated group (Fig. 1D). Notably, the expression of PrE markers SOX7 and GATA6 have been highly up-regulated in the process of $\mathrm{Cl}-\mathrm{I}$ or $\mathrm{Cl}$ induction, indicating that PrE lineage was induced (Fig. 1D). Additionally, immunostaining showed the SOX7 positive cells induced by $\mathrm{Cl}-\mathrm{I}$ or $\mathrm{Cl}$ condition further confirmed the genes expression profile (Fig. 1E). These results suggest that the combination of CHIR and IDE1 can not induce DE efficiently, but derived an MD, PrE, DE lineages, and undifferentiated hPSCs mixed population.

During the differentiation process, the dynamic expression of crucial pathway-related genes was analyzed by RT-PCR. Results showed that the expression pattern of Wnt3A and FGF8 have no significant difference among the groups. In contrast, $B M P 2$ expression was increased progressively after day 1 , 
while NODAL remains a low-level expression in cells treated with $\mathrm{Cl}-\mathrm{I}$ and $\mathrm{Cl}$ condition, compared with the CA treatment (Fig. 1F). These results suggested that lower endogenous Nodal signal and high level of BMP signal activities might be the reason why $\mathrm{Cl}-\mathrm{I}$ and $\mathrm{Cl}$ treatment yielded a mixed population. Hence, increasing the endogenous TGF- $\beta$ /Nodal signaling activity to improve current differentiation protocols potentially could be a reliable strategy for efficient DE differentiation.

\section{Manipulating small-molecule cocktails to improve DE differentiation}

Inhibition of PI3K signaling pathway or cell-cycle regulators CDK4/6 have been reported to promote the phosphorylation of Smad2/3 and regulate the signal transduction of the Nodal pathway while inducing DE differentiation $[23,24]$. Accordingly, we combined CHIR and IDE1 with the above candidate small molecules, including PI3K inhibitor LY294002 (Ly) and CDK4/6 inhibitor PD, to induce DE differentiation (Fig. 2A). During the differentiation process, morphological changes of the differentiated cells were monitored by microscopy, and phase-contrast images showed sequential morphologic changes of hPSCs differentiation into DE-like cells (Fig. S1). RT-PCR analyzed the dynamic expression of related genes, and results showed that pluripotency markers OCT4 and NANOG down-regulated significantly. In contrast, CILy and CIP induced higher DE markers SOX17 and FOXA2 expression levels than the $\mathrm{Cl}$ treated alone. Notably, the highly up-regulated expression pattern of PrE markers $S O X 7$ and GATA6 induced by $\mathrm{Cl}$ condition not seen in CILy, and CIP treated cultures (Fig. 2B). These genes expression patterns suggested that added Ly or PD could improve DE differentiation versus PrE differentiation.

Immunostaining with DE markers SOX17 and FOXA2 further confirmed that a higher proportion of DE population in CILy and CIP condition, especially CIP, efficiently generated a near homogenous SOX17 and FOXA2 co-expressed DE population, which comparable with CA treatment as a control (Fig. 2C). Further statistical analysis showed that only $64.1 \%$ of the cells co-expressing SOX17 and FOXA2 were induced by $\mathrm{Cl}$, while up to $90.2 \%, 98.5 \%$, and $99.0 \%$ in the cells induced by CILy, CIP, and CA, respectively (Fig. 2D). These results indicate that the combination of small-molecule CILy and CIP can efficiently induce DE differentiation.

\section{Elevating endogenic TGF- $\beta /$ Nodal signal to promote DE differentiation}

To test whether PD and Ly promoted the DE differentiation by regulating the transcriptional activity of TGF- $\beta /$ Nodal-Smad2/3 signaling, we examined the expression profiles of genes related to TGF- $\beta /$ Nodal, BMP, and Wnt signal pathways. Gene expression analysis showed a progressive induction of NODAL, $T G F-\beta 1$, and TGF- $\beta 2$ by CIP, comparable with the CA control group but significantly higher than $\mathrm{Cl}$ and CILy treatment. By contrast, the expressions of $B M P 2$ and $B M P 4$ were relatively lower in CIP and CA condition on the last two days of the differentiation process (Fig. $3 \mathrm{~A}$ ). These results indicate that the addition of PD could increase the endogenous TGF- $\beta /$ Nodal signal while relatively down-regulate BMP signal activity. 
On the other hand, adding Ly increased significantly and accelerated the expression of WNT3A, with the expression peak appearing two days earlier and twice higher than other induction groups (Fig. 3A). As well known that Wnt is crucial to direct hPSCs differentiation and also induce MD formation. Thus, these results aligned with the above results that CILy rapidly reduced expression of pluripotent markers OCT4 and NANOG within 1-day induction while inducing the highest level of PS/MD marker gene BRA during the differentiation and accelerated Mix/1 expression to transiently peak at day 1 (Fig. 2B). Further immunostaining showed that many BRA-positive cells were induced in CILy treatment at day 3, while few in CIP treated condition (Fig. 3B). Moreover, these CILy-derived MDs could develop into contracting cardiomyocytes in the later phase of the hepatic differentiation process (Movie.1). These results indicated that blocking the PI3K pathway with Ly amplifies the Wnt signaling, which accelerated hPSCs differentiation and MD induction.

Further analysis of protein expression by Western blot showed the effect of the different protocols on TGF- $\beta /$ Nodal signaling molecules and downstream phosphorylation level of Smad2/3. Results showed that adding PD (CIP) increased the amounts of NODAL and TGF- $\beta 1$ proteins significantly, while little change was observed in ClLy treated cells compared with $\mathrm{Cl}$ treatment. In contrast, Protein bands upon CA treated cells revealed the highest expression level. Similarly, Smad2/3 and phosphorylated Smad2/3 ( $\mathrm{p}$-Smad2 and p-Smad2) showed a low protein expression in Cl-treated cells while increased upon treatment with CIP and CA (Fig. 3C). Quantification of the protein bands verified the higher NODAL, TGF$\beta 1$ proteins, and stronger phosphorylation Smad2/3 of CIP treated samples compared to the $\mathrm{Cl}$ and CILy groups (Fig. 3D). Thus, we concluded that the addition of PD to the combination of $\mathrm{Cl}$ could up-regulate the endogenous TGF- $\beta /$ Nodal signal and the downstream Smad2/3 transduction activities to promote the differentiation of DE.

\section{Small-molecule cocktail VDF directed hepatic specification}

Further endodermal specifying a hepatic fate generally utilize growth factors including BMPs, FGFs, and HGF. To identify effective small molecules substitute growth factors for hepatic specification, we specifically explored the availability of Vc (previously used to replace BMPs in HBs expansion), the HGF agonist Dihexa, and Forskolin (FSK, an adenylate cyclase activator and thus activating CAMP/PKA signal transduction) in inducing a hepatic fate, since these small molecules have been shown to promote differentiation and proliferation of HBs in previous studies [6, 13, 25] (Fig. 4A).

We firstly induced CIP-derived DE cells into HBs, using the combination of BMP2 and FGF4 (BF). As expected, genes expression and immunostaining analyses showed the expression of HBs marker AFP and EpCAM after five days of induction (Fig. 4B, C), indicating that CIP-derived DE cells could be differentiated into HBs efficiently. Furthermore, we observed that exposure to either Dihexa or FSK in the presence of Vc resulted in induction of the hepatic marker genes AFP, HNF4a, and Albumin (ALB), with an expression rate slightly lower than the BF control group. In comparison, the triple combination of $\mathrm{Vc}$, Dihexa, and FSK (VDF) results in the best overall induction of the three hepatic markers (Fig. 4D). Further immunostaining with AFP and HNF4a confirmed that cells treated with the combination of VDF highly co- 
expressed the hepatic markers (Fig. 4E, S2), and the efficiency (95.5\%) was comparable with the BF control group (96.2\%) (Fig. 4F).

To further confirm the HBs differentiation methodology, we tested another hPSC line (iPSC line UC15) using the optimized small-molecule condition. The results showed that this iPSC line also could sequentially differentiate into DE cells and HBs, with high efficiency and expressed stages-specific markers (Fig. S3). These results indicated that the combination of small-molecule VDF could substitute growth factors to induce hepatic specification.

\section{Expansion and maintain of small-molecule derived HBs}

Our previous study has established a robust and cost-effective culture condition for generating selfrenewing HBs and functional mature HLCs, using a small-molecule cocktail in a defined culture system [13]. Based on this, we attempted to set up a complete small-molecule formula to efficiently generate expanded HBs and functional mature HLCs (Fig. 5A).

Firstly, we purified the HBs by cell sorting with EpCAM antibody, which is well known as a specific surface marker to isolate HBs (Fig. 5B). As expected, HBs populations were isolated from the differentiated cells (day 8 ) with a proportion of $95.2 \%$, which is consistent with the ratio of small-molecule VDF derived AFP and HNF4a double-positive HBs showing above. The purified HBs successfully expanded in the smallmolecule culture condition (ACDFSV) with a reliable self-renewal capacity. Additionally, the proliferative HBs could be cultured stably for at least 20 passages, without significant morphologic change and an apparent decrease in proliferative capacity (Fig. 5C).FACS analysis showed that 54.7\% EpCAM positive HBs (at passage 10) co-positive with proliferative marker Ki67 (Fig. 5D). Immunostaining further confirmed that expanding HBs almost co-expressed typical hepatic marker AFP and HNF4a. Moreover, considerable AFP-positive cells co-expressed with Ki67 (Fig. 5E), indicating the preferable proliferative capacity of HBs. Further results exhibited bipotency features in the proliferative HBs, with hepatic markers and early cholangiocyte markers SOX9 and Ep-CAM co-expression (Fig. 5E). During expansion, these cells could be frozen and thawed repeatedly.

\section{Differentiation of proliferative HBs into functional mature hepatocytes}

For hepatic maturation, the proliferative HBs then cultured in previously established small-molecule culture conditions, including A8301, Dex, Dihexa, and NH4Cl (DNAD) [13]. After five days of induction, differentiated cells displayed a homogenous and typical mature hepatocyte-like polygonal morphology (Fig. 6A). Genes expression of hepatic matured and relative functional biomarkers were analyzed to identify the derived HLCs. The results indicated that the $A L B$ and cytochrome P450 (CYP) and urea cycle enzymes transcription induced in the derived HLCs (Fig. 6B). Human PHs used as a control in the experiments. Immunostaining analyses further confirmed that the derived HLCs typically co-stained with mature hepatocyte-specific proteins ALB, A1AT, CYP3A4, and CYP2C9 (Fig. 6C). 
Furthermore, we assessed the metabolism and detoxification functions of the small-molecule derived HLCs by characterizing the activities of CYP enzymes. Three typical CYP isoforms (CYP3A4, CYP2C9, and CYP 2D6) were examined by measuring the increase in CYP isoform metabolites in response to exposure to their respective probe substrates. Results released considerable levels of CYP enzyme metabolic activities compared with that of human PHs (Fig. 6D). The HLCs also showed a high urea secretion pattern, corresponding to approximately $50 \%$ urea secreted by the human PHs (Fig. 6E). Moreover, Periodic acid-Schiff staining (PAS), indocyanine green (ICG), and LDL uptake analyses showed that HLCs displayed cytoplasmic glycogen storage, ICG, and LDL uptake abilities (Fig. 6F-H). Taken together, these results indicated that the current small-molecule protocol could direct hPSCs to efficiently differentiate, expand and maturate into metabolically functioning hepatocytes in vitro.

\section{Repopulation of mice injured liver by HBs transplantation}

To determine whether the HBs could differentiate into functional hepatocytes and repopulate the injured liver in vivo, we transplanted HBs into DMN-induced acute liver failure of NSI mice (Fig. 7A). The KaplanMeier survival estimates were determined for ten days after cell transplantation. In the sham control group, two-thirds (6 of 9) of animals died within five days after the DMN-injection while inducing acute liver failure. For the HBs transplanted groups, the survival rate was nearly $80 \%$ (7 of 9) throughout the examination period (Fig. 7B).

Hematoxylin and eosin (H\&E) staining revealed massive necrosis loci associated with inflammatory cell infiltration in the DMN treated mouse liver, suggesting DMN-induced acute liver failure. In contrast, the necrosis loci dramatically decreased and morphologically nearly restored to normal control in the HBs transplanted mice liver (Fig. 7C). The levels of AST and ALT were significantly decreased in mice transplanted with HBs compared to the sham-operated mice (Figure.7D). These results suggest that HBs transplantation benefits the parenchymal tissue and the damaged recipient liver's functional recovery.

Moreover, to trace the homing cells and analyze the repopulation efficiency, HBs were labeled with Di1 dye before transplantation. After one week of transplantation, immunostaining with a human ALB (hALB) antibody was conducted. Representative patterns of Di1 positive and hALB positive cells in the HBs transplanted mice liver were shown (Fig. 7E). In contrast, no hALB-positive cells were detected in the sham control group (Fig. 7F). These results indicated that the small-molecule protocol derived HBs could home and differentiate into mature hepatocytes after being transplanted in vivo. 4 weeks posttransplantation, Di1 and hALB co-positive cells were observed more widespread in HBs transplanted mice liver (Fig. 7G). We further analyzed the cell engraftment by the FACS examination of hALB-positive hepatocytes in the recipient's liver. Results showed that up to $14.8 \%$ of the hepatocyte mass mightily derived from the transplanted human HBs, judging by the hALB labeled cells (Fig. $7 \mathrm{H}$ ).

No teratomas or other tumor types were found in any transplanted recipients during a 4-week period. Therefore, we concluded that the current small-molecule protocol derived HBs could engraft and differentiate into functional hepatocytes, which further attains injured parenchyma tissue repopulation in the recipient's liver after transplantation. 


\section{Discussion}

This study established an efficient protocol for the specification, expansion, and maturation of hepatic cells from hPSCs, using small molecules in a chemical defined culture system. In developing the protocol, we first demonstrated that the combination of CIP could efficiently induce DE differentiation via cell cycle inhibition by PD to increase endogenous TGF- $\beta$ /Nodal signaling. Furthermore, we identified that combining Vc, Dihexa, and FSK (VDF) could substitute growth factor BMPs, FGFs, and HGF to induce hepatic specification. In addition, the small-molecule derived HBs subsequently expanded and matured into functional HLCs by the strategy established in our previous study. The generated HLCs displayed adult hepatocyte's typical functional characteristics in vitro and the ability to repopulate injured liver in vivo. Thus, the current full small-molecule based hepatic generation protocol presents an efficient and cost-effective strategy for the large-scale production of self-renewal HBs and functional mature hepatocytes for cell-based therapy and drug discovery use.

DE induction is the first stage also a critical stage of hPSCs differentiation into hepatic cells. The efficacy of DE induction and their heterogeneity influenced the following hepatic differentiation trajectory and outcome significantly $[23,26]$. Although applying a high concentration of Activin A combined with Wnt3a or Wnt agonist CHIR could efficiently differentiate hPSCs into DE $[17,19,27]$, while Activin A is an expensive growth factor, limiting its use in large-scale cell production. Small-molecule methods yielded low efficiency and reproducibility differentiation $[8,15,22,28]$, which may be due to no available small molecule that can efficiently substitute Activin A to induce DE differentiation. Melton et al. have reported that IDE1/2 can phosphorylate Smad2/3 and activate Activin A / Nodal signaling to induce hPSCs into the endodermal lineage [20]. However, IDE1 treated alone failed to efficiently generate DE in our preliminary studies (data not shown), and similar results (40-80\% SOX17-positive cells) were also reported in other studies $[7,8,22,28]$.

Further combining CHIR with IDE1 ( $\mathrm{Cl}-\mathrm{I}$ and $\mathrm{Cl}$ methods) improved the differentiation significantly but still yielded a mixture population, including $D E$, undesired $M D$ and PrE lineage, and also with undifferentiated hPSCs. Both $\mathrm{Cl}-\mathrm{I}$ and $\mathrm{Cl}$ treatment induced PrE marker SOX7 expression while barely expressed in CAinduced DE differentiation. Previously reported that low Nodal and high BMP signals would lead to the upregulation of SOX7 and PrE differentiation [29-31]. Thus, inefficient differentiation outcome may be due to insufficient endogenous Nodal signal while a high level of BMP signal activities stimulated by $\mathrm{Cl}-\mathrm{I}$ or $\mathrm{Cl}$, compared with $\mathrm{CA}$ treatment. That highlights the critical roles of endogenous signals in $\mathrm{DE}$ differentiation. Therefore, we attempted to increase endogenous TGF-b/Nodal signaling activity by manipulating the small-molecule cocktails to improve DE differentiation.

PI3K signaling pathway acted as a threshold at the initial stage of hPSCs differentiation [23, 32, 33]. The activation and transduction of Nodal and Wnt signaling pathways require decreased PI3K activity to trigger hPSCs differentiation [33]. Similarly, cell-cycle regulators CDK4/6 also have been reported to regulate the phosphorylation of Smad2/3 and control the signal transduction of the Activin A/Nodal pathway [24]. Moreover, inhibiting PI3K or CDK4/6 with small molecules promoted Smad2/3 to bind and 
activate DE genes while inducing DE differentiation. Hence, we combined $\mathrm{Cl}$ with candidate small molecules, including PI3K inhibitor LY and CDK4/6 inhibitor PD, to induce DE differentiation.

Our finding demonstrated that adding PD to $\mathrm{Cl}$ treatment (CIP) improved DE gene expression while dismissing SOX7 upregulation, resulting in efficient DE formation. Further results released that adding PD increased endogenous TGF- $\beta$ superfamily genes and proteins (Nodal and TGF- $\beta 1$ ) expression significantly while downregulating the transcription of $B M P 2$ and $B M P 4$. Furthermore, the addition of PD also increased the phosphorylation of Smad2/3 ( $\mathrm{p}-\mathrm{Smad} 2$ and p-Smad2), which is comparable with CA treatment. These results aligned with previous reports that PD could induce endogenous Nodal signaling in hPSCs differentiation [24]. Therefore, we concluded that adding PD to $\mathrm{Cl}$ can up-regulate endogenous TGF- $\beta /$ Nodal signaling and downstream Smad2/3 transduction activities while relatively down-regulating BMP signal activity, which leads to the promotion of DE fate versus MD/PrE differentiation.

Moreover, we found that the addition of Ly to Cl treatment (CILy) led to exceeding amplification of Wnt signal, consistent with previously reported that PI3K negatively regulates the activity of the Wnt signal pathway [33, 34]. However, high Wnt signal activity tends to specify a ME formation [35-37], thereby affecting the differentiation of DE. This might account for why CILy treatment led to the highest MD marker gene BRA level and induced BRA-positive MD lineages during the differentiation process while yielding lower efficiency DE formation.

Small molecules have been identified to promote DE induction, while few chemicals have been reported to support hepatic specification effectively. Further specifies DE to a hepatic fate in previous smallmolecule based protocols mainly relied on sequential treatment with sodium butyrate and DMSO, which played a part in epigenetic modifications and were associated with ambiguous hepatic induction $[15,17$, 38]. However, variable studies reported whether these nonspecific agents are sufficient for hepatic differentiation. Rambhatla et al. indicated that DMSO was insufficient to differentiate DE cells into hepatic lineage [39]. In addition, both DMSO and sodium butyrate inhibits cell growth, which might compromise the yield of hESCs derived hepatocytes for applications. Yet, no report indicated effective small molecules substitute for BMPs and FGFs to direct hepatic specification efficiently. Thus, identifying novel small molecules with hepatic-specific induction would contribute to the strategy of functional hepatic cells production.

Our recent study has identified that Vc, FSK, and Dihexa could replace BMP4, EGF, and HGF, respectively, supporting HBs expansion and maintenance [13]. Also, we demonstrated that Vc could phosphorylate BMP downstream Smad1/5/8, consistent with the previous report that Vc serves a similar effect in cardiomyogenesis [40]. Besides, FSK has been demonstrated essential for downregulating EMT marker gene expression and sustaining PHs gene expression [25]. Moreover, Dihexa was identified in a screen that assessed the capacity to potentiate the biological activity of HGF and can act as a potent HGF agonist $[6,41]$, which could mimic the function of HGF in HBs differentiation and expansion. These results suggested the potential of Vc, Dihexa, and FSK to substitute growth factors for hepatic specification. In the present study, we showed that treatment with Vc and FSK combined with Dihexa 
(VDF) resulted in high expression of hepatic genes and proteins (AFP and HNF4a), comparable with growth factors treated group as control. These results indicated that the combination of small-molecule VDF could substitute BMPs, FGFs, and HGF to induce hepatic specification efficiently.

HBs have capable of self-renewal and potency to differentiate into hepatocytes. Hence expanding HBs, especially in small-molecule culture conditions to scalable expand HBs derived from hPSCs would be an ideal strategy for efficient, large-scale, and cost-effective generating hepatic cells for the potential clinical application [12, 42]. According to this view, our previous study has established a robust and cost-effective small-molecule culture condition for the generation of self-renewing HBs and functional mature HLCs [13]. Thus, we then adopted the previously established culture condition to expand the small-molecule derived HBs. Our finding released that the purified HBs could successfully expand in the proliferative culture condition (ACDFSV), with a reliable self-renewal capacity and hepatic properties maintained. Additionally, the proliferative HBs could undergo long-term culture for at least 20 passages without significant morphologic change and an obvious decrease in their proliferative capacity.

Moreover, the proliferative HBs could be further efficiently differentiated into functional mature HLCs after being induced with mature conditions (DNAD). The small-molecule derived HLCs expressed various representative markers and exhibited typical function activities. Remarkably, the metabolic activity of CYP enzymes was comparable to PHs. In addition, after being transplanted into NSI mice with acute liver failure, these hepatic cells could engraft and differentiate into hepatocytes, resulting in considerable liver repopulation and functional recovery. These results indicated that our small-molecule based hepatic induction method induces acquired hepatocytes to function in vitro and in vivo.

\section{Conclusions}

In summary, we have developed a robust and cost-effective strategy for inducing hPSCs to proliferative HBs and functional hepatocytes using the small-molecule protocol, which could serve as a powerful foundation for future studies to define culture conditions using GMP-compatible products. Furthermore, this work provides a novel, cost-effective way for generating large-scale and functional human hepatic cells, which would result in an invaluable impact in the cell-based therapy industry.

\section{Abbreviations}

hPSCs

Human pluripotent stem cells

HBs

Hepatoblasts

HLCs

Hepatocyte-like cells

$\mathrm{PHs}$

Primary hepatocytes 
DE

Definitive endoderm

Primitive endoderm

PrE

NSI mice

NOD-SCID-IL2RG ${ }^{-/-}$mice

$\mathrm{CHIR}$

CHIR99021

CYP

Cytochrome P450

Indocyanine green

ICG

Periodic acid-Schiff staining

PAS.

\section{Declarations}

\section{Acknowledgements}

The authors thank the National Basic Research Program of China and Guangdong Province Science and Technology Plan for the funding support.

\section{Funding}

This study was supported by the National Key R\&D Program of China (2019YFA0111300, 2018YFA0108200, 2018YFC1106400), the Sino-German rapid response funding call for COVID-19 related research (C-0031), Frontier Research Program of Bioland Laboratory (Guangzhou Regenerative Medicine and Health Guangdong Laboratory) (2018GZR110105011), National Natural Science Foundation of China $(31871379,22075127,31972926)$, the Natural Science Foundation of Guangdong Province (2018A030313128, 2018A030313214), and the Guangdong Key Research and Development Plan (2019B020234003), Guangzhou Science and technology planning project (202102020988).

\section{Authors' contributions}

TP, NW, and JZ contributed equally to this work. TP wrote the manuscript; JZ, NW, YC, YZ, YX and JF revised the manuscript; TP performed the experiments and analyzed the data; YC performed the animal experiments and function assays of hepatocytes; $F Y, X L, Y L, S L, K L$ and KY assisted with the experiments and analyzed the data; YL and YG directed the study, critical revised and final approval of the manuscript.

\section{Ethics approval and consent to participate}


All procedures were conducted with the approval of the ethics committee of GIBH and in accordance with the Animal Welfare \& Ethics Committee of GIBH.

\section{Consent for publication}

Not applicable.

\section{Availability of data and material}

The datasets supporting the conclusions of this article are included within the article.

\section{Compliance with ethics guidelines}

The authors declare that they have no competing interests.

\section{References}

1. Q. Li, A.P. Hutchins, Y. Chen, S. Li, Y. Shan, B. Liao, D. Zheng, X. Shi, Y. Li, W.Y. Chan, G. Pan, S. Wei, X. Shu, D. Pei, A sequential EMT-MET mechanism drives the differentiation of human embryonic stem cells towards hepatocytes, Nat Commun 8 (2017) 15166.

2. L.T. Ang, A.K.Y. Tan, M.I. Autio, S.H. Goh, S.H. Choo, K.L. Lee, J. Tan, B. Pan, J.J.H. Lee, J.J. Lum, C.Y.Y. Lim, I.K.X. Yeo, C.J.Y. Wong, M. Liu, J.L.L. Oh, C.P.L. Chia, C.H. Loh, A. Chen, Q. Chen, I.L. Weissman, K.M. Loh, B. Lim, A Roadmap for Human Liver Differentiation from Pluripotent Stem Cells, Cell Rep 22(8) (2018) 2190-2205.

3. S.J. Mun, J.S. Ryu, M.O. Lee, Y.S. Son, S.J. Oh, H.S. Cho, M.Y. Son, D.S. Kim, S.J. Kim, H.J. Yoo, H.J. Lee, J. Kim, C.R. Jung, K.S. Chung, M.J. Son, Generation of expandable human pluripotent stem cellderived hepatocyte-like liver organoids, Journal of hepatology 71(5) (2019) 970-985.

4. S. Chen, J. Wang, H. Ren, Y. Liu, C. Xiang, C. Li, S. Lu, Y. Shi, H. Deng, X. Shi, Hepatic spheroids derived from human induced pluripotent stem cells in bio-artificial liver rescue porcine acute liver failure, Cell research 30(1) (2020) 95-97.

5. S. Feng, J. Wu, W.L. Qiu, L. Yang, X. Deng, Y. Zhou, Y. Chen, X. Li, L. Yu, H. Li, Z.R. Xu, Y. Xiao, X. Ren, L. Zhang, C. Wang, Z. Sun, J. Wang, X. Ding, Y. Chen, P. Gadue, G. Pan, M. Ogawa, S. Ogawa, J. Na, P. Zhang, L. Hui, H. Yin, L. Chen, C.R. Xu, X. Cheng, Large-scale Generation of Functional and Transplantable Hepatocytes and Cholangiocytes from Human Endoderm Stem Cells, Cell Rep 33(10) (2020) 108455.

6. R. Siller, S. Greenhough, E. Naumovska, G.J. Sullivan, Small-molecule-driven hepatocyte differentiation of human pluripotent stem cells, Stem cell reports 4(5) (2015) 939-52.

7. F. Tasnim, D. Phan, Y.C. Toh, H. Yu, Cost-effective differentiation of hepatocyte-like cells from human pluripotent stem cells using small molecules, Biomaterials 70 (2015) 115-25.

8. M.S. Bogacheva, S. Khan, L.K. Kanninen, M. Yliperttula, A.W. Leung, Y.R. Lou, Differences in definitive endoderm induction approaches using growth factors and small molecules, Journal of cellular 
physiology 233(4) (2018) 3578-3589.

9. T. Katsuda, M. Kawamata, K. Hagiwara, R.U. Takahashi, Y. Yamamoto, F.D. Camargo, T. Ochiya, YACConversion of Terminally Committed Hepatocytes to Culturable Bipotent Progenitor Cells with Regenerative Capacity, Cell stem cell 20(1) (2017) 41-55.

10. A. Korostylev, P.U. Mahaddalkar, O. Keminer, K. Hadian, K. Schorpp, P. Gribbon, H. Lickert, A highcontent small molecule screen identifies novel inducers of definitive endoderm, Molecular metabolism 6(7) (2017) 640-650.

11. Yohan Kim, Kyojin Kang, Seung Bum Lee, Daekwan Seo, Sangtae Yoon, Sung Joo Kim, Kiseok Jang, Yun Kyung Jung, Kyeong Geun Lee, Valentina M Factor, Jaemin Jeong, Dongho Choi, Small molecule-mediated reprogramming of human hepatocytes into bipotent progenitor cells. Journal of Hepatolgy. 2019 Jan;70(1):97-107.

12. T. Pan, Y. Chen, Y. Zhuang, F. Yang, Y. Xu, J. Tao, K. You, N. Wang, Y. Wu, X. Lin, F. Wu, Y. Liu, Y. Li, G. Wang, Y.-x. Li, Synergistic modulation of signaling pathways to expand and maintain the bipotency of human hepatoblasts, Stem cell research \& therapy 10(1) (2019).

13. T. Pan, J. Tao, Y. Chen, J. Zhang, A. Getachew, Y. Zhuang, N. Wang, Y. Xu, S. Tan, J. Fang, F. Yang, X. Lin, K. You, Y. Gao, Y.X. Li, Robust expansion and functional maturation of human hepatoblasts by chemical strategy, Stem cell research \& therapy 12(1) (2021) 151.

14. F.Z. Asumda, K.E. Hatzistergos, D.M. Dykxhoorn, S. Jakubski, J. Edwards, E. Thomas, E.R. Schiff, Differentiation of hepatocyte-like cells from human pluripotent stem cells using small molecules, Differentiation 101 (2018) 16-24.

15. C. Du, Y. Feng, D. Qiu, Y. Xu, M. Pang, N. Cai, A.P. Xiang, Q. Zhang, Highly efficient and expedited hepatic differentiation from human pluripotent stem cells by pure small-molecule cocktails, Stem cell research \& therapy 9(1) (2018) 58.

16. S. Mathapati, R. Siller, A.A.R. Impellizzeri, M. Lycke, K. Vegheim, R. Almaas, G.J. Sullivan, SmallMolecule-Directed Hepatocyte-Like Cell Differentiation of Human Pluripotent Stem Cells, Current Protocols in Stem Cell Biology 2016, pp. 1G.6.1-1G.6.18.

17. Z. Li, J. Wu, L. Wang, W. Han, J. Yu, X. Liu, Y. Wang, Y. Zhang, G. Feng, W. Li, G.N. Stacey, Q. Gu, B. Hu, L. Wang, Q. Zhou, J. Hao, Generation of qualified clinical-grade functional hepatocytes from human embryonic stem cells in chemically defined conditions, Cell death \& disease 10(10) (2019) 763.

18. G. Lee, H. Kim, J.Y. Park, G. Kim, J. Han, S. Chung, J.H. Yang, J.S. Jeon, D.H. Woo, C. Han, S.K. Kim, H.J. Park, J.H. Kim, Generation of uniform liver spheroids from human pluripotent stem cells for imaging-based drug toxicity analysis, Biomaterials 269 (2021) 120529.

19. J. Liu, T. Pan, Y. Chen, Y. Liu, F. Yang, Q. Chen, N. Abbas, M. Zhong, Q. Zhang, Y. Xu, Y.X. Li, Repair of acute liver damage with immune evasive hESC derived hepato-blasts, Stem cell research 49 (2020) 102010.

20. M. Borowiak, R. Maehr, S. Chen, A.E. Chen, W. Tang, J.L. Fox, S.L. Schreiber, D.A. Melton, Small molecules efficiently direct endodermal differentiation of mouse and human embryonic stem cells, Cell stem cell 4(4) (2009) 348-58. 
21. U. Diekmann, O. Naujok, R. Blasczyk, T. Muller, Embryonic stem cells of the non-human primate Callithrix jacchus can be differentiated into definitive endoderm by Activin-A but not IDE-1/2, J Tissue Eng Regen Med 9(4) (2015) 473-9.

22. Y. Tahamtani, M. Azarnia, A. Farrokhi, A. Sharifi-Zarchi, N. Aghdami, H. Baharvand, Treatment of human embryonic stem cells with different combinations of priming and inducing factors toward definitive endoderm, Stem cells and development 22(9) (2013) 1419-32.

23. Kyle M. Loh, Lay T. Ang, J. Zhang, V. Kumar, J. Ang, Jun Q. Auyeong, Kian L. Lee, Siew H. Choo, Christina Y.Y. Lim, M. Nichane, J. Tan, Monireh S. Noghabi, L. Azzola, Elizabeth S. Ng, J. DurruthyDurruthy, V. Sebastiano, L. Poellinger, Andrew G. Elefanty, Edouard G. Stanley, Q. Chen, S. Prabhakar, Irving L. Weissman, B. Lim, Efficient Endoderm Induction from Human Pluripotent Stem Cells by Logically Directing Signals Controlling Lineage Bifurcations, Cell stem cell 14(2) (2014) 237-252.

24. S. Pauklin, L. Vallier, The cell-cycle state of stem cells determines cell fate propensity, Cell 155(1) (2013) 135-47.

25. D.Y. Xiang C, Meng G, Soon Yi L, Sun S, Song N, Zhang X, Xiao Y, Wang J, Yi Z, Liu Y, Xie B, Wu M, Shu J, Sun D, Jia J, Liang Z, Sun D, Huang Y, Shi Y, Xu J, Lu F, Li C, Xiang K, Yuan Z, Lu S, Deng H, Long-term functional maintenance of primary human hepatocytes in vitro, Science 364(6438) (2019) 399-402.

26. L. Yiangou, A.D.B. Ross, K.J. Goh, L. Vallier, Human Pluripotent Stem Cell-Derived Endoderm for Modeling Development and Clinical Applications, Cell stem cell 22(4) (2018) 485-499.

27. A. Sahabian, J. Dahlmann, U. Martin, R. Olmer, Production and cryopreservation of definitive endoderm from human pluripotent stem cells under defined and scalable culture conditions, $\mathrm{Nat}$ Protoc 16(3) (2021) 1581-1599.

28. E. Hoveizi, M. Nabiuni, K. Parivar, J. Ai, M. Massumi, Definitive endoderm differentiation of humaninduced pluripotent stem cells using signaling molecules and IDE1 in three-dimensional polymer scaffold, J Biomed Mater Res A 102(11) (2014) 4027-36.

29. K.A. D'Amour, A.D. Agulnick, S. Eliazer, O.G. Kelly, E. Kroon, E.E. Baetge, Efficient differentiation of human embryonic stem cells to definitive endoderm, Nature Biotechnology 23(12) (2005) 15341541.

30. K. Takayama, M. Inamura, K. Kawabata, K. Tashiro, K. Katayama, F. Sakurai, T. Hayakawa, M.K. Furue, $\mathrm{H}$. Mizuguchi, Efficient and directive generation of two distinct endoderm lineages from human ESCs and iPSCs by differentiation stage-specific SOX17 transduction, PloS one 6(7) (2011) e21780.

31. S. Sudheer, R. Bhushan, B. Fauler, H. Lehrach, J. Adjaye, FGF inhibition directs BMP4-mediated differentiation of human embryonic stem cells to syncytiotrophoblast, Stem cells and development $21(16)$ (2012) 2987-3000.

32. L. Sui, L. Bouwens, J.K. Mfopou, Signaling pathways during maintenance and definitive endoderm differentiation of embryonic stem cells, The International journal of developmental biology 57(1) (2013) 1-12. 
33. Amar M. Singh, D. Reynolds, T. Cliff, S. Ohtsuka, Alexa L. Mattheyses, Y. Sun, L. Menendez, M. Kulik, S. Dalton, Signaling Network Crosstalk in Human Pluripotent Cells: A Smad2/3-Regulated Switch that Controls the Balance between Self-Renewal and Differentiation, Cell stem cell 10(3) (2012) 312326.

34. T.S. Huang, L. Li, L. Moalim-Nour, D. Jia, J. Bai, Z. Yao, S.A. Bennett, D. Figeys, L. Wang, A Regulatory Network Involving beta-Catenin, e-Cadherin, PI3k/Akt, and Slug Balances Self-Renewal and Differentiation of Human Pluripotent Stem Cells In Response to Wnt Signaling, Stem Cells 33(5) (2015) 1419-33.

35. X. Lian, C. Hsiao, G. Wilson, K. Zhu, L.B. Hazeltine, S.M. Azarin, K.K. Raval, J. Zhang, T.J. Kamp, S.P. Palecek, Robust cardiomyocyte differentiation from human pluripotent stem cells via temporal modulation of canonical Wnt signaling, Proceedings of the National Academy of Sciences of the United States of America 109(27) (2012) E1848-57.

36. U. Kreuser, J. Buchert, A. Haase, W. Richter, S. Diederichs, Initial WNT/ $\beta$-Catenin Activation Enhanced Mesoderm Commitment, Extracellular Matrix Expression, Cell Aggregation and Cartilage Tissue Yield From Induced Pluripotent Stem Cells, Frontiers in cell and developmental biology 8 (2020).

37. Paul W Burridge, Elena Matsa, Praveen Shukla, Ziliang C Lin, Jared M Churko, Antje D Ebert, Feng Lan, Sebastian Diecke, Bruno Huber, Nicholas M Mordwinkin, Jordan R Plews, Oscar J Abilez, Bianxiao Cui, Joseph D Gold, Joseph C Wu, chemically defined generation of human cardiomyocytes. Nature Methods. 2014 Aug;11(8):855-60.

38. K. Czysz, S. Minger, N. Thomas, DMSO efficiently down regulates pluripotency genes in human embryonic stem cells during definitive endoderm derivation and increases the proficiency of hepatic differentiation, PloS one 10(2) (2015) e0117689.

39. C.C. Rambhatla L, Kundu P, Peng Y, Carpenter MK, Generation of Hepatocyte-Like Cells from Human Embryonic Stem Cells, Cell Transplant (2003).

40. M.G. Perino, S. Yamanaka, D.R. Riordon, Y. Tarasova, K.R. Boheler, Ascorbic acid promotes cardiomyogenesis through SMAD1 signaling in differentiating mouse embryonic stem cells, PloS one 12(12) (2017) e0188569.

41. A.T. McCoy, C.C. Benoist, J.W. Wright, L.H. Kawas, J.M. Bule-Ghogare, M. Zhu, S.M. Appleyard, G.A. Wayman, J.W. Harding, Evaluation of metabolically stabilized angiotensin IV analogs as procognitive/antidementia agents, J Pharmacol Exp Ther344(1) (2013) 141-54.

42. T. Touboul, S. Chen, C.C. To, S. Mora-Castilla, K. Sabatini, R.H. Tukey, L.C. Laurent, Stage-specific regulation of the WNT/beta-catenin pathway enhances differentiation of hESCs into hepatocytes, Journal of hepatology 64(6) (2016) 1315-26.

\section{Figures}


A

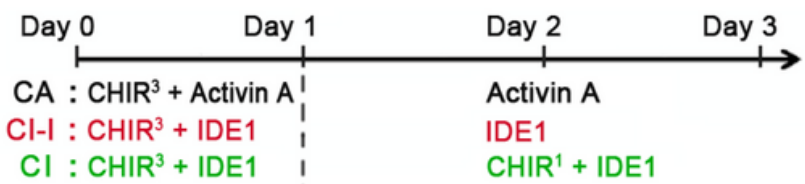

$\mathrm{B}$

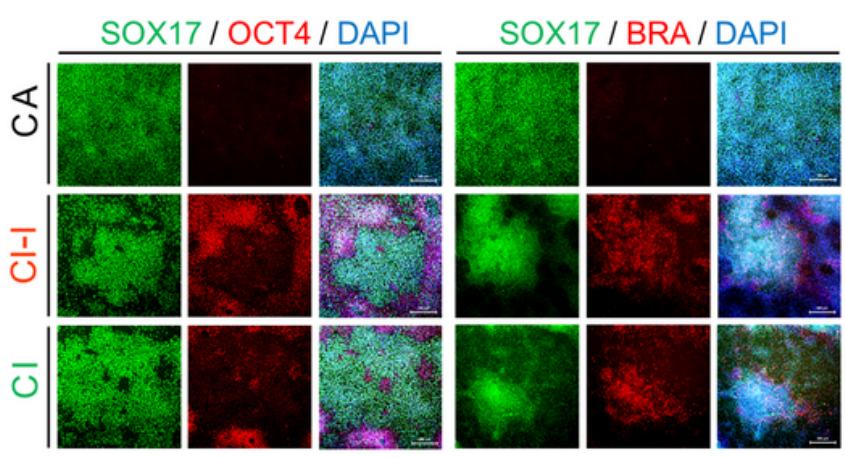

C

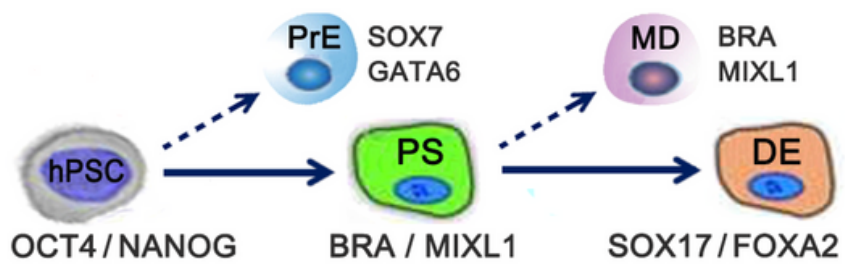

D
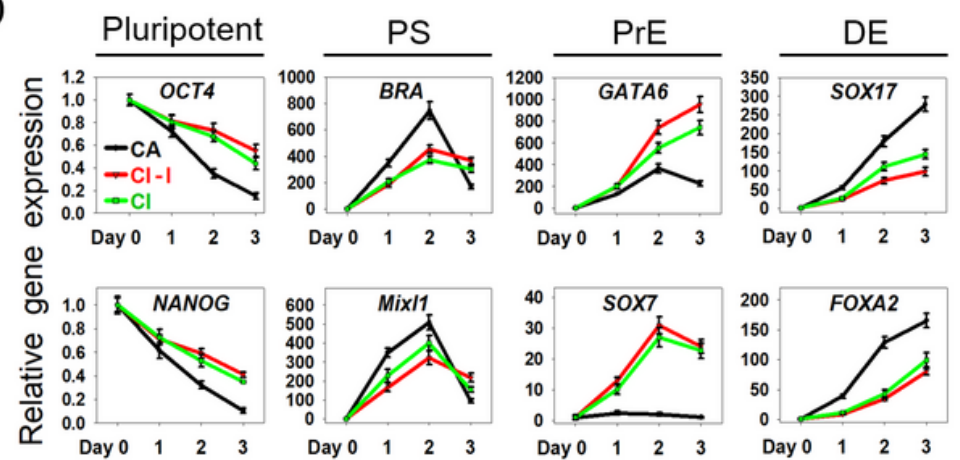

E

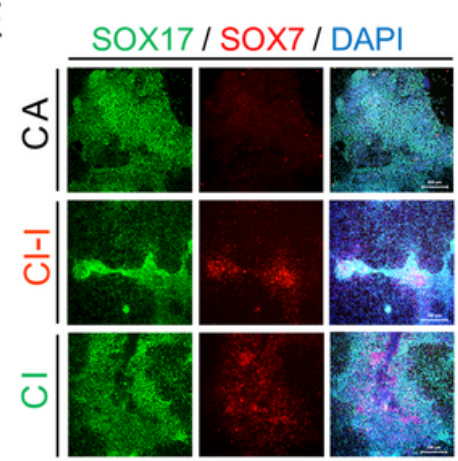

$\mathrm{F}$

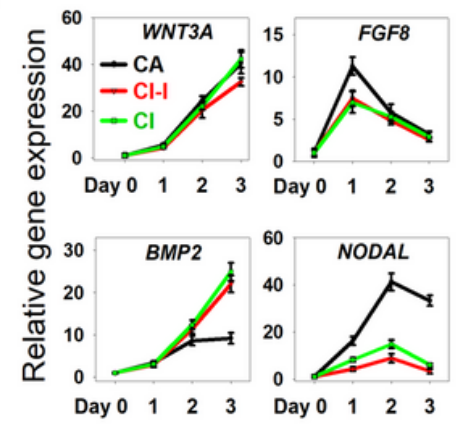

Figure 1

\section{CHIR and IDE1 inefficiently induced DE differentiation from hPSCs}

(A) Schematic of the two-step chemical strategy to differentiate hPSCs into DE cells. CHIR ${ }^{3}: 3 \mu \mathrm{M}$ CHIR99021, CHIR ${ }^{1}$ : $1 \mu \mathrm{M}$ CHIR99021. (B) Immunostaining analyzed SOX17, OCT4, and BRA expression in the differentiated cells. Scale bars $200 \mu \mathrm{m}$. (C) Overview of lineage relationships during DE differentiation from hPSCs. PS: primitive streak, PrE: primitive endoderm, MD: mesoderm, DE: definitive endoderm. (D) Quantitative RT-PCR analysis lineage relative genes expression of differentiated cells during DE induction. Data are presented as mean \pm SEM, $n=3$. (E) Immunostaining analyzed SOX17 and SOX7 expression in the differentiated cells. Scale bars $200 \mu \mathrm{m}$. (F) Quantitative RT-PCR analyzed signaling pathway-related genes expression patterns in DE induction with different protocols. Data are presented as mean $\pm S E M, n$ $=3$. 
A

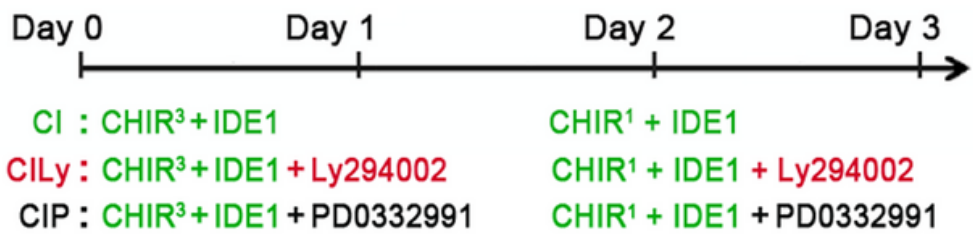

B
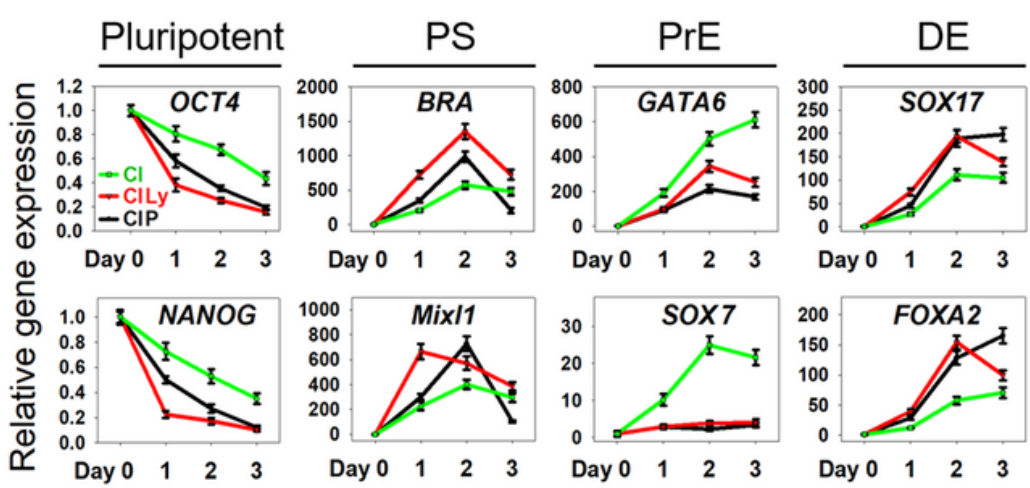

$\mathrm{D}$

\begin{tabular}{|c|c|c|c|c|}
\hline Treatment & Cl & CILy & CIP & CA \\
\hline FOXA2 $^{+}$ & $64.6 \%+/-3.8$ & $90.5 \%+/-6.5$ & $98.9 \%+/-7.2$ & $99.2 \%+/-5.6$ \\
\hline SOX17 & $78.4 \%+/-5.2$ & $96.5 \%+/-7.4$ & $99.2 \%+/-6.6$ & $99.5 \%+/-6.2$ \\
\hline FOXA2 $^{+} /$SOX17 & $64.1 \%+/-4.8$ & $90.2 \%+/-6.2$ & $98.5 \%+/-6.5$ & $99.0 \%+/-5.5$ \\
\hline
\end{tabular}
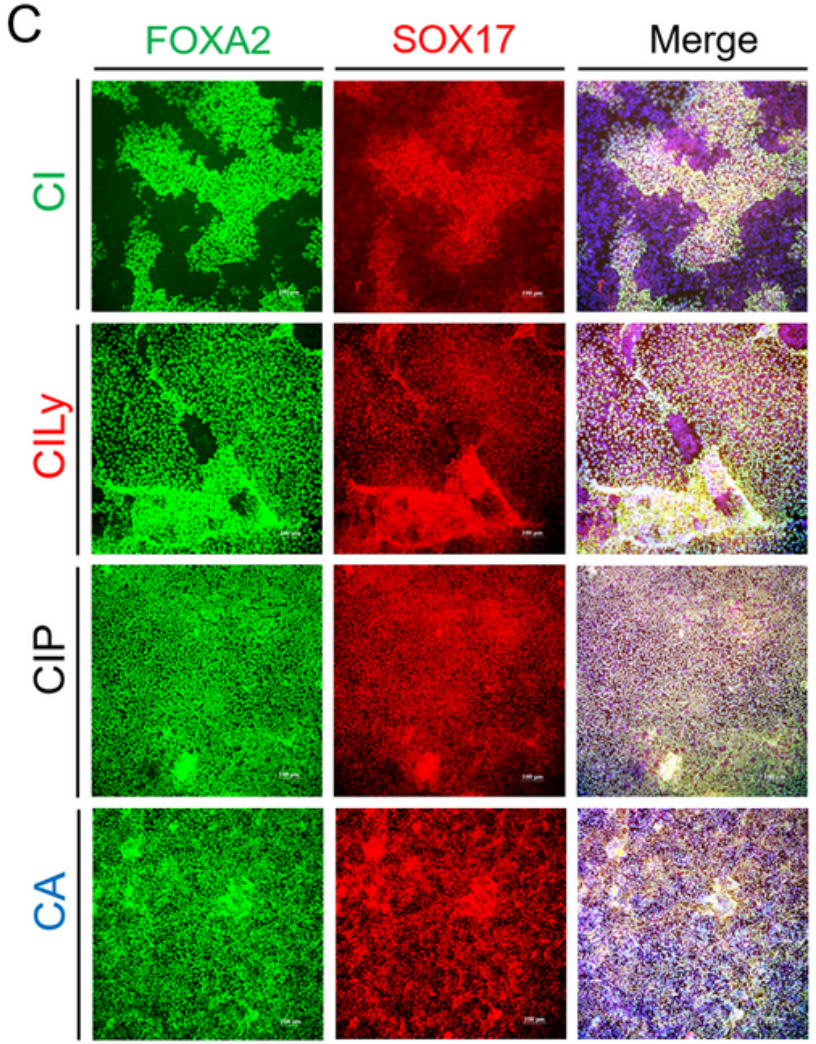

Figure 2

\section{Manipulating small-molecule cocktails to improve DE differentiation}

(A) Schematic of the strategy to differentiate hPSCs into DE cells with small molecules. (B) Quantitative RT-PCR analysis lineage relative genes expression of differentiated cells during DE induction with different protocols. Data are presented as mean $\pm S E M, n=3$. (C) Immunostaining analyses of $S 0 X 17$ and FOXA2 expression in DE cells derived from different protocols. Scale bars $100 \mu \mathrm{m}$. (D) The efficiency of SOX17 and FOXA2 expression after different cocktails treatment, determined by counting positive cells. Efficiencies are presented as the percentage of positive cells plus or minus the SD of all fields counted. 
A

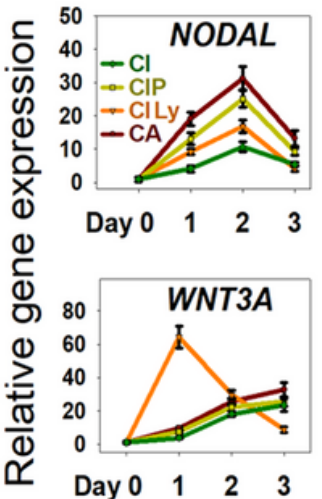

B
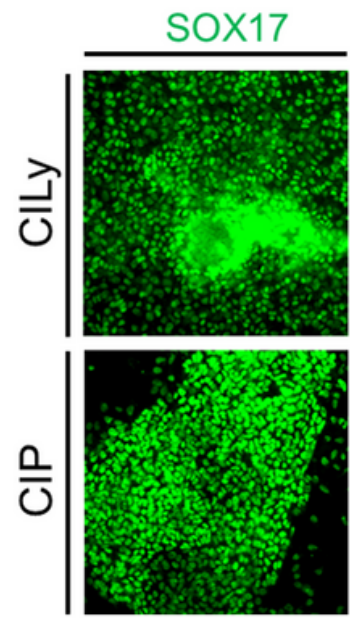
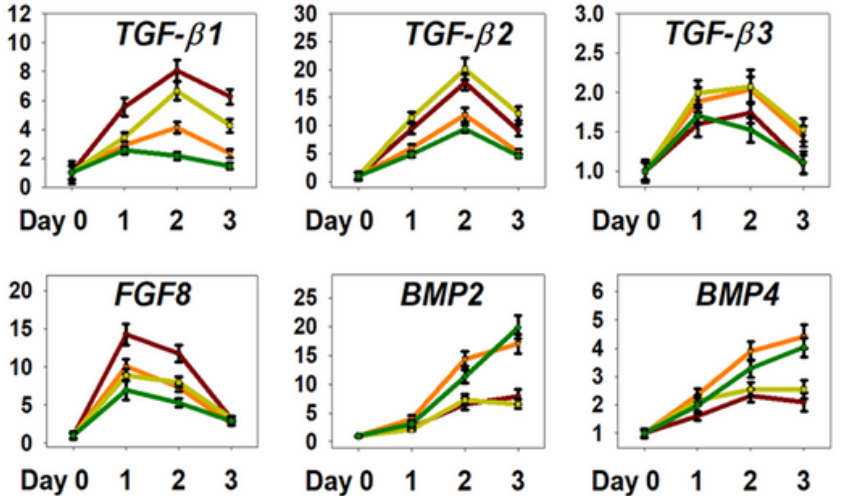

Merge
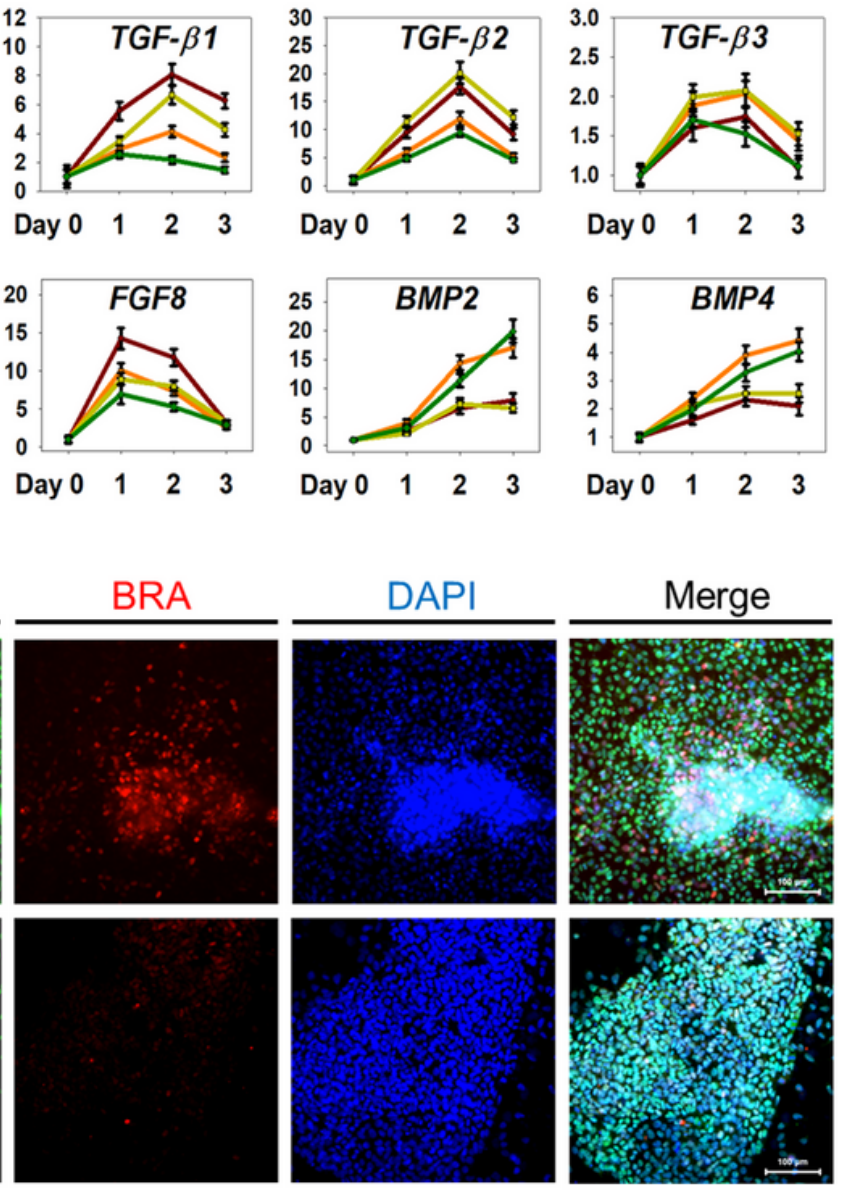

C

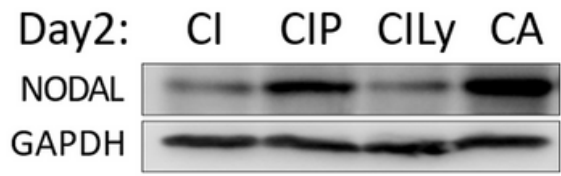

TGF- $\beta 1$

GAPDH

p-Smad2

Smad2

p-Smad3

Smad3

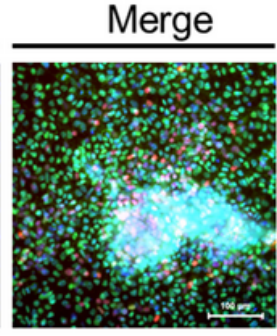

$\mathrm{D}$
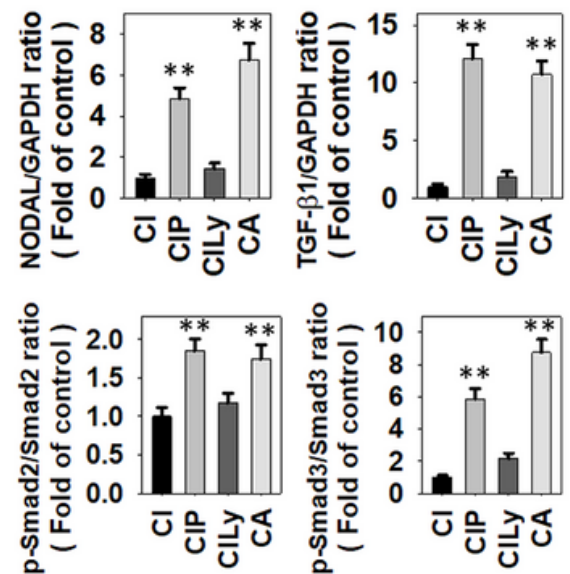

Figure 3

\section{Elevating endogenic TGF- $\beta /$ Nodal signal to promote DE differentiation}

(A) Quantitative RT-PCR analyzed signaling pathway-related genes expression patterns in DE induction with different protocols. Data are presented as mean $\pm S E M, n=3$. (B) Immunostaining analyzed SOX17 and BRA expression in the CILy and CIP derived differentiated cells. Scale bars $100 \mu \mathrm{m}$. (C)

Representative immunoblots of NODAL, TGF- $\beta 1$, and the phosphorylation of Smad2/3 at day 2 during DE induction with different protocols. (D) Quantification of the protein expression levels. Data were expressed as mean $\pm \mathrm{SD}, \mathrm{n}=3$ for each group, $* P<0.05$, $* \star P<0.01$ vs control (Cl) group. 


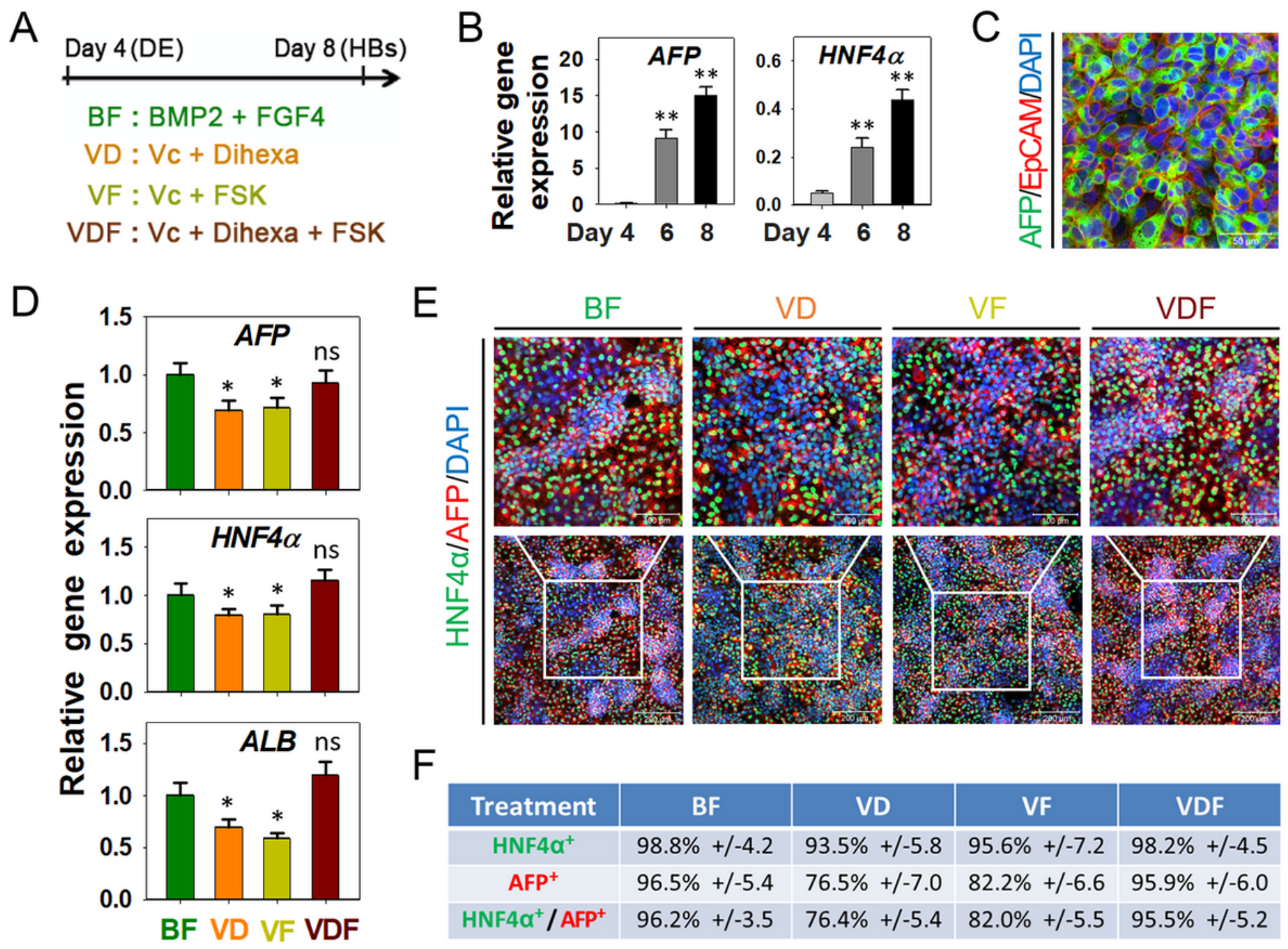

Figure 4

\section{Small-molecule cocktail VDF directed hepatic specification}

(A) Schematic of the strategy to identify chemical culture cocktails for HB specification. (B) RT-PCR analyzed AFP and HNF4a expression during hepatic specification. Data are presented as mean \pm SEM, $\mathrm{n}=3$. (C) Immunostaining analyses of AFP and HNF4a expansion after 5 days of BF induction. Scale bars $100 \mu \mathrm{m}$. (D) RT-PCR results show hepatic genes expression in differentiated cells induced by different small molecule cocktails. Data are presented as mean $\pm \mathrm{SEM}, \mathrm{n}=3$. (E) Immunostaining analyses of AFP and HNF4a expression after different small molecule cocktails induced. Scale bars $100 \mu \mathrm{m}$. (F) The efficiency of AFP and HNF4a expression after different chemical cocktails treatment, determined by counting positive cells. Efficiencies are presented as the percentage of positive cells plus or minus the SD of all fields counted. 
A

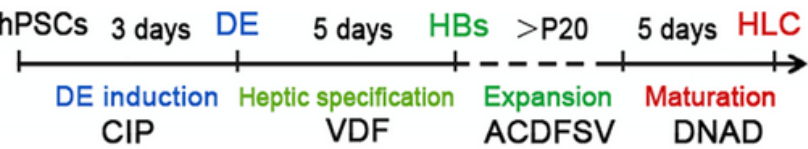

$\mathrm{B}$

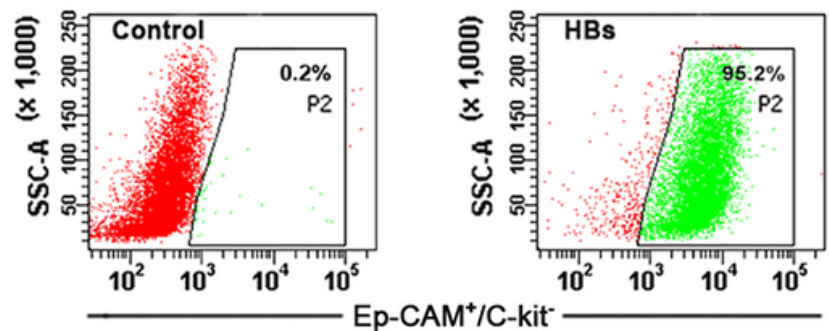

D

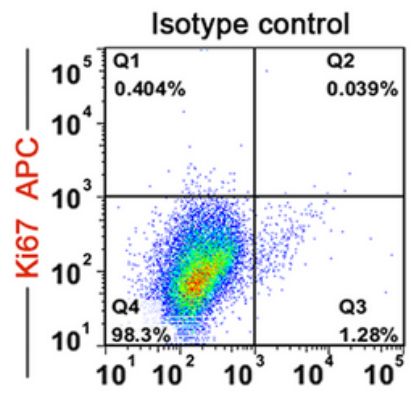

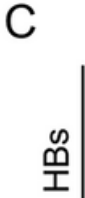

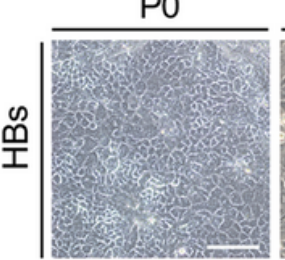
P10
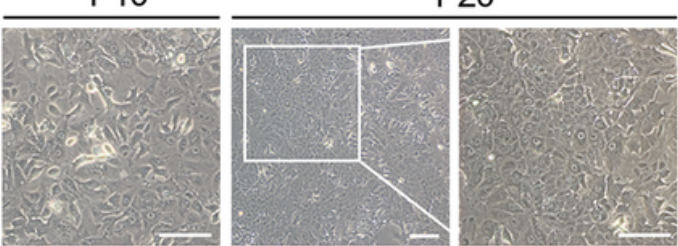

E
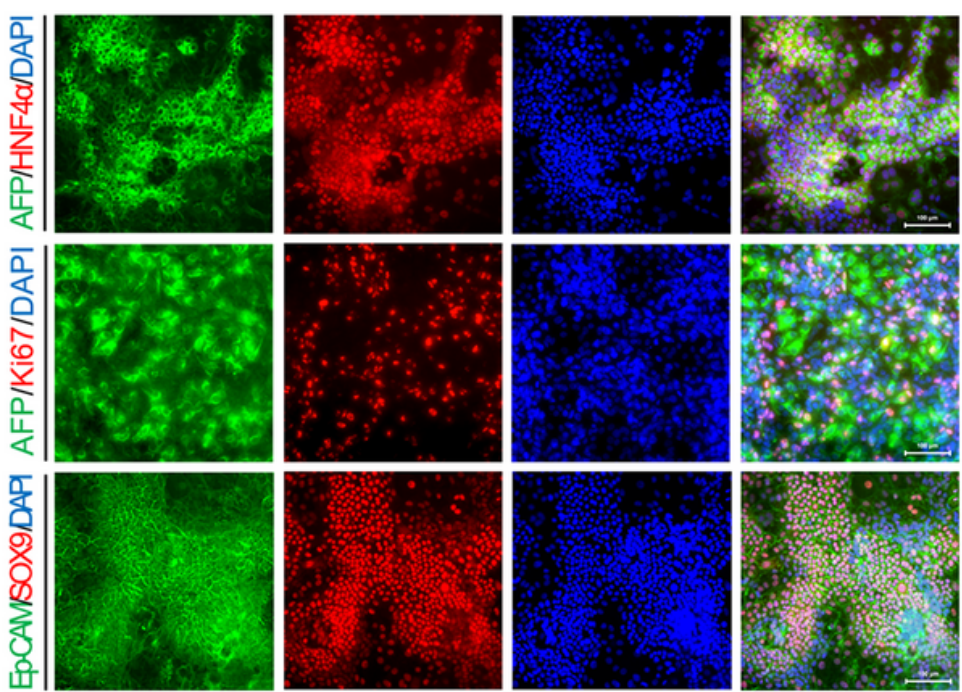

\section{Figure 5}

\section{Expansion and maintain of small-molecule derived HBs}

(A) A schematic description of stepwise differentiates hPSCs into HBs and HLCs. (B) Flow cytometric sorting of E-pCAM ${ }^{+}$HBs from day 8 differentiated cells. (C) Phase-contrast images of expanding HBs. Scale bar $100 \mu \mathrm{m}$. (D) Flow cytometric analyses of E-pCAM and Ki67 expression in expanded HBs (passage 20). (E) Immunostaining analyses of HBs marker proteins on expanded HBs. Scale bars 100 $\mu \mathrm{m}$. 
A

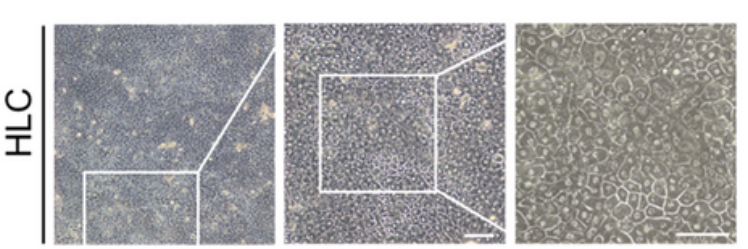

C
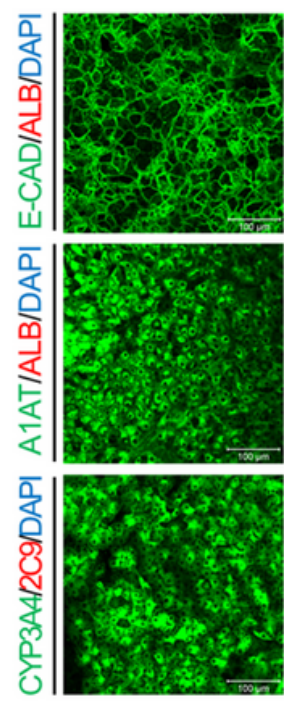

B
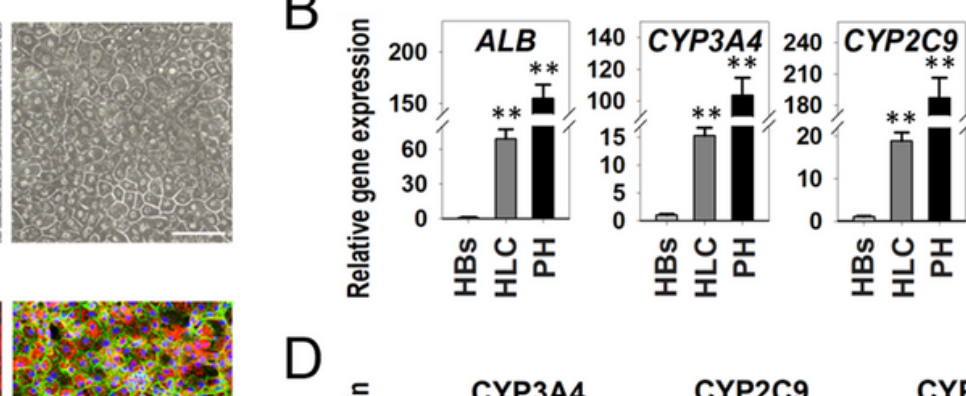

$D$

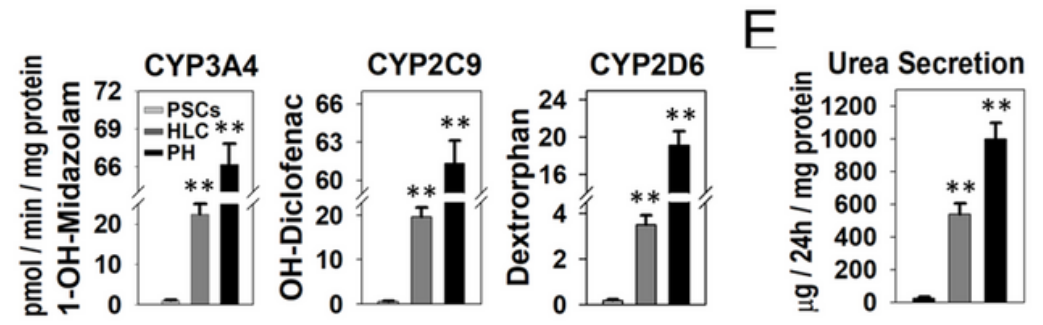

$\mathrm{F}$

G

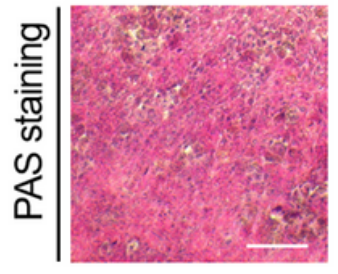

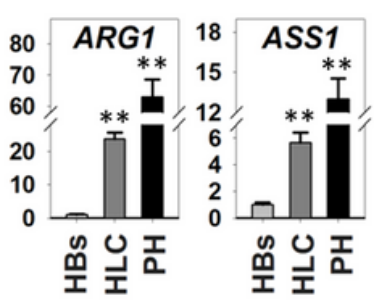

$\mathrm{H}$
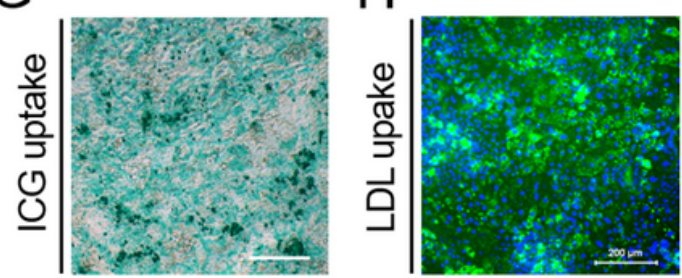

Figure 6

\section{Differentiation of proliferative HBs into functional hepatocytes}

(A) The morphology of small-molecule protocol derived HLCs. HLCs exhibited polygonal shapes and distinct round nuclei; even some had two nuclei. Scale bar $100 \mu \mathrm{m}$. (B) Quantitative RT-PCR analyzed the genes expression levels of ALB, the CYP and urea cycle enzymes. Gene expression was normalized to HBs. Data are presented as mean $\pm \mathrm{SEM}, \mathrm{n}=3 .{ }^{*} P<0.05$, $* \star P<0.01$. (C) Immunostaining analyses of mature hepatocyte markers (ALB, A1AT, CYP3A4, and CYP2C9) on small-molecule protocol derived HLCs. Scale bars $100 \mu \mathrm{m}$. (D) CYP450 activity assay of HLCs. Data are presented as mean \pm SEM, $n=3$. * $P<0.05$, $\star * P<0.01$. (E) Urea secretion of HLCs was analyzed. Data are presented as mean $\pm \mathrm{SEM}, \mathrm{n}=3$. * $P<0.05$, $\star * P<0.01$. (F) PAS staining on HLCs. Scale bar represents $100 \mu \mathrm{m}$. (G) ICG uptake analyses in HLCs. Scale bar represents $100 \mu \mathrm{m}$. (H) The HLCs were stained by Alexa-Flour 488-ac-LDL. Scale bar 200 $\mu \mathrm{M}$. 
A

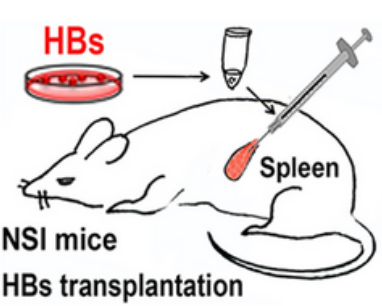

B

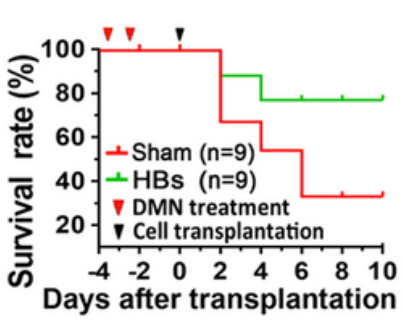

D
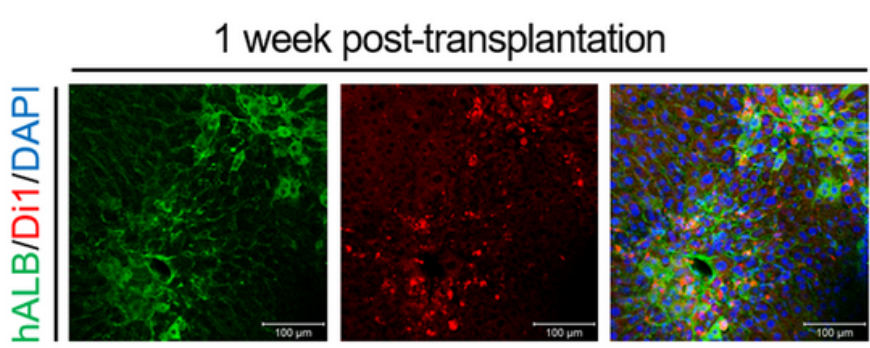

G
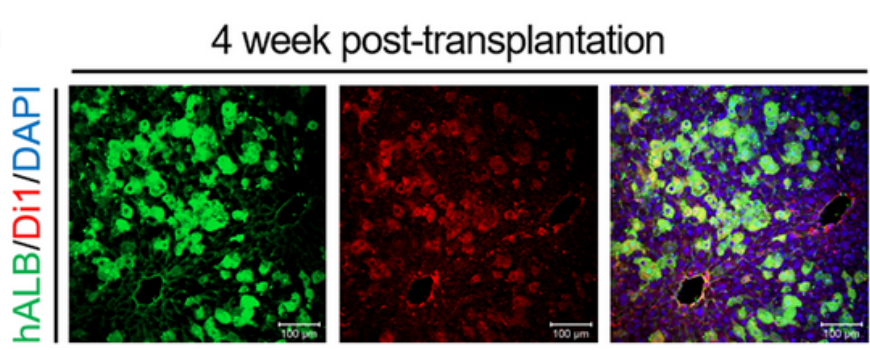
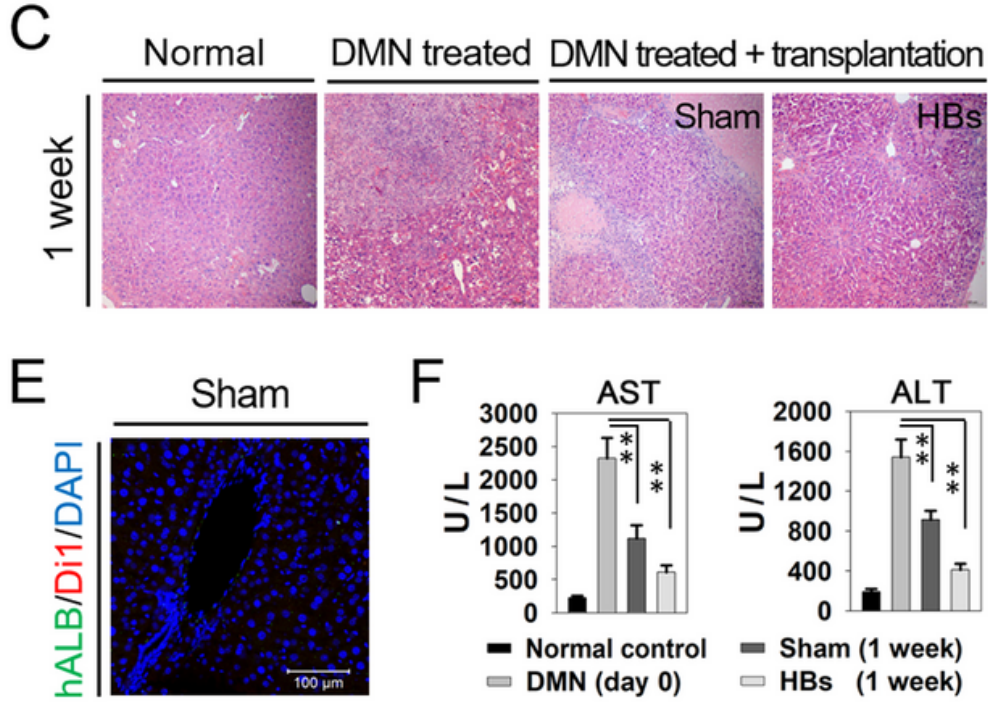

- Normal control - Sham (1 week)

$\square$ DMN (day 0) $\square$ HBs (1 week)

$\mathrm{H}$

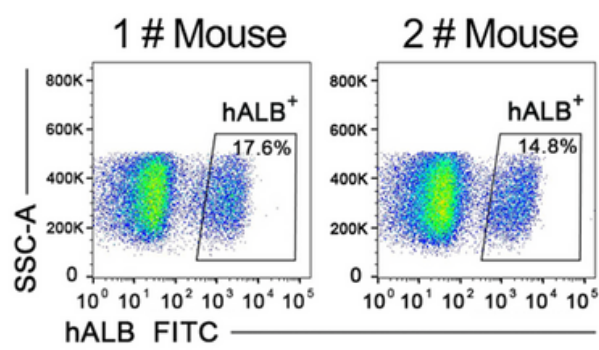

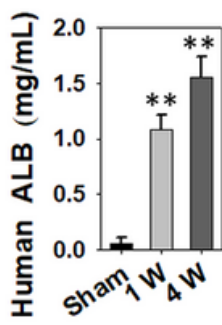

Figure 7

\section{Repopulation of mice injured liver by HBs transplantation}

(A) Schematic diagram depicting HBs transplantation experimental schedule in immune-deficiency mice

(B) Survival curve of mice. (C) Hematoxylin and eosin staining in mice liver. (D-E) Engraftment of transplanted human HBs in mice liver after 1 week transplantation, as indicated by immunostaining of human ALB (green) and Di1 (red). Sham mice's liver as the negative control. (F) Detection of AST and ALT levels in mice serum at one week after HBs transplantation. Data were analyzed by 2-tailed t tests. (G) After 4 weeks of cell transplantation, the reproduction of mice liver is indicated by immunostaining of human ALB (green) and Di1 (red). (H) Flow cytometric analyses human ALB ${ }^{+}$hepatocytes in mice liver transplanted with human HBs. (I) Human ALB secretion in the mice serum. Data are presented as mean \pm SEM, $n=4$.

\section{Supplementary Files}

This is a list of supplementary files associated with this preprint. Click to download.

- Movie.1.mp4

- SupplementaryMaterial.docx 